\title{
Novel Characterization of Drug-associated Pancreatitis in Children
}

\author{
Harrison X. Bai ${ }^{\star}$, Michael H. Ma ${ }^{\star}$, Abrahim I. Orabi ${ }^{\star}$, Alexander Park ${ }^{\dagger}$, Sahibzada U. Latif ${ }^{\ddagger}$, \\ Vineet Bhandari", and Sohail Z. Husain ${ }^{*}$ \\ "Department of Pediatrics, Yale University School of Medicine, New Haven, CT \\ tDepartment of Internal Medicine, University of Washington, Seattle \\ ‡Department of Internal Medicine, University of Alabama, Birmingham
}

\begin{abstract}
Background and Objectives-Medications are a major cause of acute pancreatitis; however, little is known about their influence in children. Our primary aims were to identify common comorbidities and concomitant pancreatitis etiologies in children with drug-associated pancreatitis. Our secondary aims were to identify the most commonly associated drugs in the different age groups, evaluate management practices, and compare drug-associated cases with non-drug-associated cases.
\end{abstract}

Patients and Methods-In the present study, we examined children (ages 0-20 years) admitted to Yale-New Haven Children's Hospital with pancreatitis between 1994 and 2007.

Results-Of a total of 271 pediatric cases, drugs were associated with pancreatitis in $25.6 \%$ (55). The 3 most common comorbidities in children with drug-associated pancreatitis were seizure disorders, acute lymphocytic leukemia, and Crohn disease. One third of drug-associated cases had an additional pancreatitis etiology. The most commonly associated drugs were valproic acid and corticosteroids. Compared with non-drug-associated cases, children with drug-associated cases were more likely to undergo CT scanning $(54.5 \%$ vs $28.4 \% ; P<0.001)$, stay in the hospital longer (10 vs 4 days; $P<0.001$ ), and transition to parenteral nutrition from a nil per os status $(37.5 \%$ vs $21.2 \% ; P<0.05)$. There was a higher frequency of valproic acid-associated cases in children younger than 11 years (29.4\% vs $9.5 \%$ in the 11 - to 20 -year-old age group).

Conclusions-Our study underscores the importance of considering drugs as a cause and a contributor to pancreatitis in children, particularly valproic acid in young children.

\section{Keywords}

acute pancreatitis; children; drug; medications; pediatric; valproic acid

Acute pancreatitis is a painful, inflammatory disorder that has multiple associated etiologies, including common bile duct stones, medications, systemic disease, trauma, infections, metabolic disorders, and hereditary causes $(1,2)$. It accounts for $>200,000$ annual hospital

Copyright $@ 2011$ by European Society for Pediatric Gastroenterology, Hepatology, and Nutrition and North American Society for Pediatric Gastroenterology, Hepatology, and Nutrition

Address correspondence and reprint requests to Sohail Z. Husain, Department of Pediatrics, 333 Cedar St, PO Box 208064, New Haven, CT 06520 (sohail.husain@yale.edu).

The authors report no conflicts of interest. 
admissions and thus ranks as the third most common inpatient gastrointestinal diagnosis in the United States $(3,4)$.

Among the common etiologies, little is known about medication-associated pancreatitis in children. In a previous study that characterized a cohort of 215 children with pancreatitis admitted during a 12-year span, we found that drugs were associated with pancreatitis in about one-quarter of cases (1). The most common medications were valproic acid (13\%), prednisone (12\%), and mesalamine (9\%) (1).

In the present study, our primary aims were to identify common comorbidities and concomitant pancreatitis etiologies in children with drug-associated pancreatitis. Our secondary aims were to identify the most commonly associated drugs in the different age groups and evaluate management practices in this cohort with respect to the overall group.

\section{PATIENTS AND METHODS}

\section{Study Group}

Upon institutional review board approval, we obtained data on all of the children (ages 0-20 years) admitted to Yale-New Haven Children's Hospital with pancreatitis between 1994 and 2007. Details of the cohort, including referral trends for pancreatitis over time, etiologies, and ethnic breakdown, have been recently published. About one-quarter of the patients had $>1$ etiology identified (1).

\section{Inclusion Criteria}

The inclusion criteria for acute pancreatitis in children were as previously described (1): briefly, serum amylase or lipase elevated $>3$ times the upper limit of normal; radiographic evidence consistent with acute pancreatitis (demonstrating a minimum of pancreatic parenchymal abnormalities or peripancreatic fluid) on ultrasound or computed tomography (CT); serum lipase >1.5 times the upper limit of normal (without a nonpancreatic cause for hyperlipasemia); and the presence of 2 of the clinical features of acute pancreatitis (abdominal pain or irritability, nausea or vomiting, and epigastric tenderness). Cases were excluded if they had chronic pancreatitis documented on CT or endoscopic retrograde cholangiopancreatography (ERCP) or if they had an incomplete chart record.

Medication-related pancreatitis etiology was assigned only if the patient was taking a medication listed in the 2007 American Gastroenterological Association Technical Bulletin on Acute Pancreatitis as having "definite," "probable," or "possible" association with acute pancreatitis (5). In addition, the medication had to be taken actively before the onset of acute pancreatitis.

\section{Data Collection}

Relevant clinical data were collected directly from patients' charts. Notable fields included patient demographics, body mass index percentile, weight-for-age percentile, clinical presentation, hospital management, and clinical course of pancreatitis.

\section{Statistical Analysis}

Statistical analysis was performed by comparing continuous variables using a Student $t$ test or a Mann-Whitney $U$ test, whereas discrete variables were compared using $\chi^{2}$ analysis. A $P$ value $<0.05$ denoted statistical significance. Data were compiled and analyzed with SPSS (SPSS Inc, Chicago, IL) software. Age-adjusted percentiles for body mass index and weightfor-age were calculated with SAS (SAS Institute, Cary, NC). 


\section{RESULTS}

There were 594 cases identified by International Classification of Disease- 9 codes for pancreatitis. Of these, $271(45.6 \%)$ met inclusion criteria, comprising 215 children. We found 55 episodes that were drug associated, corresponding to 51 patients. The demographic characteristics and comorbidities of the drug-associated cohort are provided in Tables 1 and 2 , respectively. A subcomparison of younger children ( $0-10$ years) with older children (1120 years) showed no differences in age-adjusted weight characteristics or ethnic breakdown. Twice as many children with drug-associated pancreatitis had additional comorbidities compared with non-drug-associated cases $(89 \%$ vs $39 \%, P<0.0001)$. The most common comorbidities in the drug-associated cases were seizure disorders (20\%), acute lymphocytic leukemia (20\%), and Crohn disease (11\%). One third of patients with drug-associated pancreatitis had an additional etiology attributable to pancreatitis (Fig. 1). The most common additional etiology was classified as systemic illness, followed by biliary pancreatitis, structural defects of the pancreas, viral infection, hypertriglyceridemia, and cystic fibrosis. Using a list of medications associated with pancreatitis derived from a 2007 American Gastroenterological Association technical bulletin, 17 medications were identified in our cohort. Ten were in the "definite," 4 in the "probable," and 2 in the "possible" category for association with pancreatitis. Steroids were the most common drug association, followed by valproic acid, mesalamine, and trimethoprim/sulfamethoxazole (Fig. 2). Although most patients had only 1 drug association $(\mathrm{n}=35,63.6 \%), 29 \%$ had 2 drugs identified, and 4 cases (7.3\%) had 3 or 4 drugs identified.

There was no statistically significant difference in demographic characteristics and clinical presentation between drug-associated and non-drug-associated groups (Table 3). Most children in our larger cohort with acute pancreatitis underwent some form of radiographic evaluation to establish a diagnosis or examine etiologies (Table 4). The most common radiographic modalities performed were ultrasound, CT, ERCP, and magnetic resonance cholangiopancreatography. Patients with a drug-associated etiology received twice as many CT scans $(P=0.0002)$ but fewer ERCPs $(P=0.006)$ than those with a non-drug-associated etiology.

Hospital trends including median length of hospital stay, rate of gastroenterology (GI) consultation, and modes of nutrition were examined (Table 5). The drug-associated patients stayed in the hospital longer $(P=0.0004)$ and were more likely to transition to parenteral nutrition from a nil per os status $(P=0.02)$. There was a higher frequency of valproic acid use in the younger age group (0-10 years) than the older age group (11-20 years) in drugassociated cases (Fig. 3). This finding was even more prominent in cases that had no additional etiology (Fig. 4). There was no difference in the distribution of medications between drug-associated cases with a concomitant etiology and those without a concomitant etiology (data not shown).

\section{DISCUSSION}

In this 13-year analysis of 271 cases of acute pancreatitis in children, we report 3 key comparisons between drug-associated pancreatitis and other etiologies. Patients with drugassociated pancreatitis are more likely to receive CT scans but less likely to undergo ERCP than those with a non-drug-associated etiology, stay in the hospital longer, and receive parenteral nutrition during oral feeding.

To our knowledge, no study has examined pancreatitis with a drug-associated etiology in children, although several studies have characterized this particular etiology in patients of all ages. Vinklerova et al (6) retrospectively examined 170 cases of pancreatitis and identified 9 
cases of drug-induced acute pancreatitis and compared these cases to the total acute pancreatitis cohort. Azathioprine was found to be the most frequent causative agent. Eland et al (7) characterized 33 cases of drug-associated acute pancreatitis. In another retrospective study, Lankisch et al (8) evaluated the incidence of drug-induced pancreatitis by a questionnaire survey of 45 German centers of gastroenterology. The incidence of druginduced acute pancreatitis was only $1.4 \%$ in the present study, compared with $25.6 \%$ in our study. The reason for this discrepancy could be that we categorized drug-associated pancreatitis even in the presence of a concomitant etiology of pancreatitis.

We studied the concordance of comorbidities in children with drug-associated pancreatitis and found that seizure disorder, acute lymphocytic leukemia, and Crohn disease were the 3 most common comorbidities. It also was observed that about half of these patients (children and adults) with drug-associated pancreatitis had either a hematologic abnormality or Crohn disease (influenced by referral pattern). The high prevalence of these disorders with drugassociated pancreatitis could be accounted for by 2 major factors. First, many of the drugs are given to actually treat those disorders; for example, a child with acute lymphocytic leukemia may have received L-asparaginase, 6-mercaptopurine, and corticosteroids, and similarly, a child with Crohn disease may be taking the latter 2 drugs. Thus, the presence of the comorbidity may be a complete confounder and may have no bearing on pancreatitis. Alternatively, comorbidities such as Crohn disease may be independent risk factors for pancreatitis because of the higher likelihood of gallstones, anatomic abnormalities of the duodenum, or innate immunologic dyscrasia (9). For instance, patients with Crohn disease often have autoimmune pancreatitis type 2 (10). The only way to know whether the drug and comorbidity have an additive or even synergistic effect on the development of pancreatitis would be to design a large cohort study comparing pancreatitis risk in these well-matched groups: patients using the drug of interest who have the comorbidity; drug users who lack the comorbidity; and patients who have the comorbidity but have not used the drugs. Our findings underscore the need for such future studies.

Another interesting finding was that valproic acid-associated pancreatitis cases were more frequent among younger children. One explanation for this is that valproic acid is the drug of choice for absence seizures, an epileptic disorder primarily of young children $(11,12)$. Thus, the increased frequency of pancreatitis may merely reflect greater usage of the drug in this younger age group. Another potential reason is that there may be developmental differences between younger and older children that predispose the former to drug-induced pancreatitis with valproic acid. Notwithstanding the age differences, valproic acid is a drug associated commonly with pancreatitis. The World Health Organization reported that among 2749 reports of drug-associated acute pancreatitis between 1968 and 1993, valproic acid $(8 \%, \mathrm{n}=219)$ was the most frequently reported drug (12). In all of the case reports of valproic acid-induced acute pancreatitis, 75\% occurred in the pediatric population (13). Three children with valproic acid-associated pancreatitis were shown to develop recurrent disease with rechallenge, suggesting causality $(12,14,15)$. In a case series of 11 children who had valproic acid-induced acute pancreatitis, children with a history of drug allergies with rashes were at increased risk, whereas serum drug level, treatment duration, and presence of concomitant antiepileptic drugs were not considered risk factors (16). Our findings highlight the importance of considering valproic acid-associated pancreatitis, particularly in young children.

We found that children with drug-associated pancreatitis were twice as likely to have CT scans ordered and were more likely to transition to parenteral nutrition rather than oral feedings. It is possible that they were perceived to be sicker than the patients with non-drugassociated pancreatitis, especially because they had a greater frequency of comorbidities $(89 \%$ vs $39 \%)$. 
The limitations of our study include those inherent in its retrospective nature and the fact that all of our cases of pancreatitis were mild. Even though a strength of the study is that we examined each patient chart manually to ensure that inclusion criteria for acute pancreatitis were met, exclusion of a large number of patients from the initial coding identification could be a limitation because of selection bias. It was also impossible to establish causality because this would require rechallenging patients after stopping the drug. Physician awareness of associations with certain drugs such as valproic acid or certain diseases such as acute lymphocytic leukemia may increase the likelihood of testing routinely or more promptly and hence lead to ascertainment bias toward increased cases. Furthermore, it is possible that referral bias may make the association of drugs to pancreatitis less applicable to nontertiary care settings. Although our findings certainly require further validation, they emphasize a recurrent theme in the approach to any child who presents with pancreatitis: a thorough history with exploration of present medications is necessary. Medications listed in the American Gastroenterological Association technical bulletin and implicated by our study should be considered carefully as potential contributors to pancreatitis and withheld if feasible. In summary, drug-associated pancreatitis in children is common. Drug-associated cases received twice as many CT scans, stayed in the hospital longer, and were more likely to transition to parenteral nutrition from a nil per os status. Larger prospective studies are required to better understand this etiology in children.

\section{Acknowledgments}

The present study was supported by a Children's Digestive Health and Nutrition Young Investigator Award (to S.Z.H.).

\section{References}

1. Park A, Latif SU, Shah AU, et al. Changing referral trends of acute pancreatitis in children: a 12year single-center analysis. J Pediatr Gastroenterol Nutr. 2009; 49:316-22. [PubMed: 19503003]

2. Bai HX, Lowe ME, Husain SZ. What have we learned about acute pancreatitis in children? J Pediatr Gastroenterol Nutr. 2011; 52:262-70. [PubMed: 21336157]

3. Wu BU, Conwell DL. Acute pancreatitis part I: approach to early management. Clin Gastroenterol Hepatol. 2010; 8:410-6. [PubMed: 19896558]

4. Everhart JE, Ruhl CE. Burden of digestive diseases in the United States part III: liver, biliary tract, and pancreas. Gastroenterology. 2009; 136:1134-44. [PubMed: 19245868]

5. Forsmark CE, Baillie J. AGA Institute technical review on acute pancreatitis. Gastroenterology. 2007; 132:2022-44. [PubMed: 17484894]

6. Vinklerova I, Prochazka M, Prochazka V, et al. Incidence, severity, and etiology of drug-induced acute pancreatitis. Dig Dis Sci. 2010; 55:2977-81. [PubMed: 20499176]

7. Eland IA, van Puijenbroek EP, Sturkenboom MJ, et al. Drug-associated acute pancreatitis: twentyone years of spontaneous reporting in The Netherlands. Am J Gastroenterol. 1999; 94:2417-22. [PubMed: 10484002]

8. Lankisch PG, Droge M, Gottesleben F. Drug induced acute pancreatitis: incidence and severity. Gut. 1995; 37:565-7. [PubMed: 7489946]

9. Pitchumoni CS, Rubin A, Das K. Pancreatitis in inflammatory bowel diseases. J Clin Gastroenterol. 2010; 44:246-53. [PubMed: 20087199]

10. Maire F, Le Baleur Y, Rebours V, et al. Outcome of patients with type 1 or 2 autoimmune pancreatitis. Am J Gastroenterol. 2011; 106:151-6. [PubMed: 20736934]

11. Hughes JR. Absence seizures: a review of recent reports with new concepts. Epilepsy Behav. 2009; 15:404-12. [PubMed: 19632158]

12. Coulter DL, Allen RJ. Pancreatitis associated with valproic acid therapy for epilepsy. Ann Neurol. 1980; 7:92. [PubMed: 6767434] 
13. Badalov N, Baradarian R, Iswara K, et al. Drug-induced acute pancreatitis: an evidence-based review. Clin Gastroenterol Hepatol. 2007; 5:648-61. [PubMed: 17395548]

14. Camfield PR, Bagnell P, Camfield CS, et al. Pancreatitis due to valproic acid. Lancet. 1979; 1:1198-9. [PubMed: 86928]

15. Fecik SE, Stoner SC, Raphael J, et al. Recurrent acute pancreatitis associated with valproic acid use for mood stabilization. J Clin Psychopharmacol. 1999; 19:483-4. [PubMed: 10505598]

16. Sinclair DB, Berg M, Breault R. Valproic acid-induced pancreatitis in childhood epilepsy: case series and review. J Child Neurol. 2004; 19:498-502. [PubMed: 15526953] 


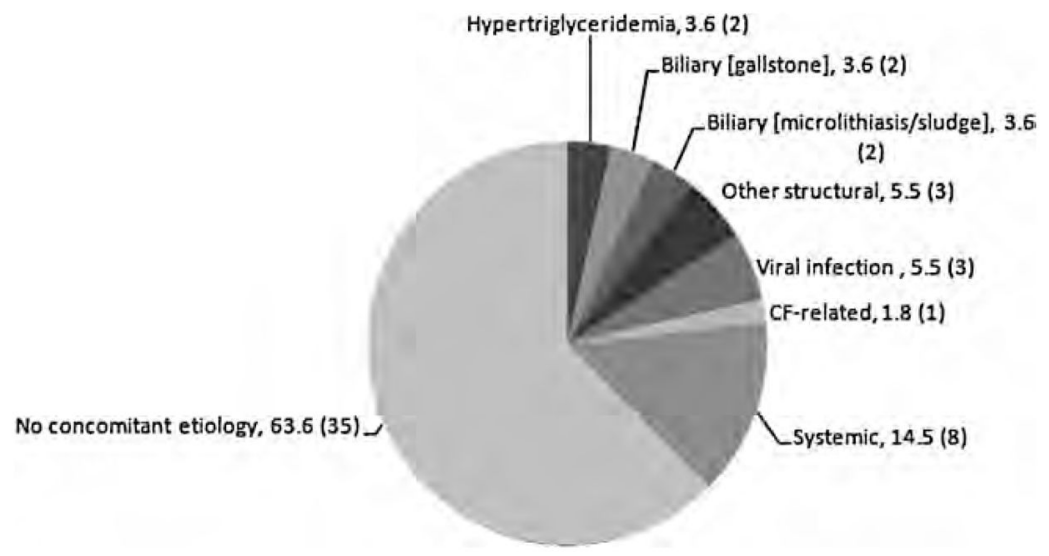

FIGURE 1.

Concomitant etiologies identified in childhood cases of drug-associated pancreatitis, \% (n). $\mathrm{CF}=$ cystic fibrosis. 


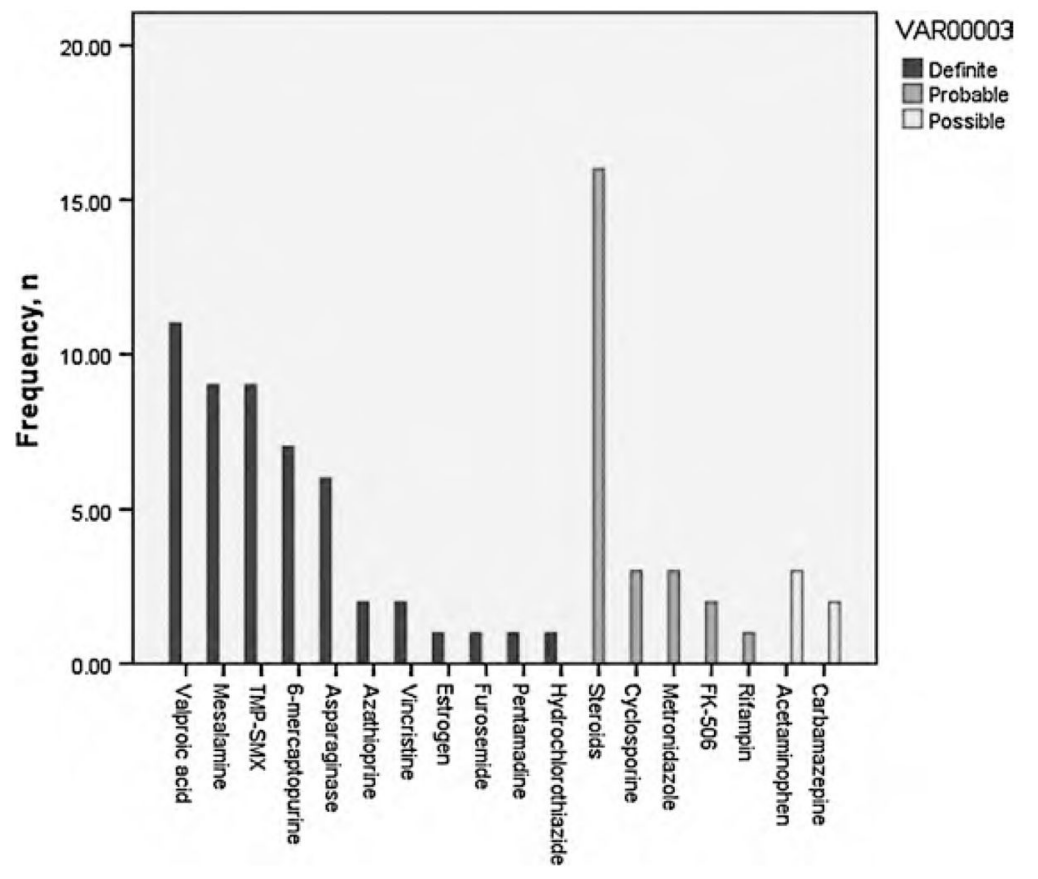

FIGURE 2.

Frequency of drugs associated with acute pancreatitis in children, organized according to the 2007 American Gastroenterological Association technical bulletin of risk associations (5). TMP-SMX = trimethoprim/sulfamethoxazole. 


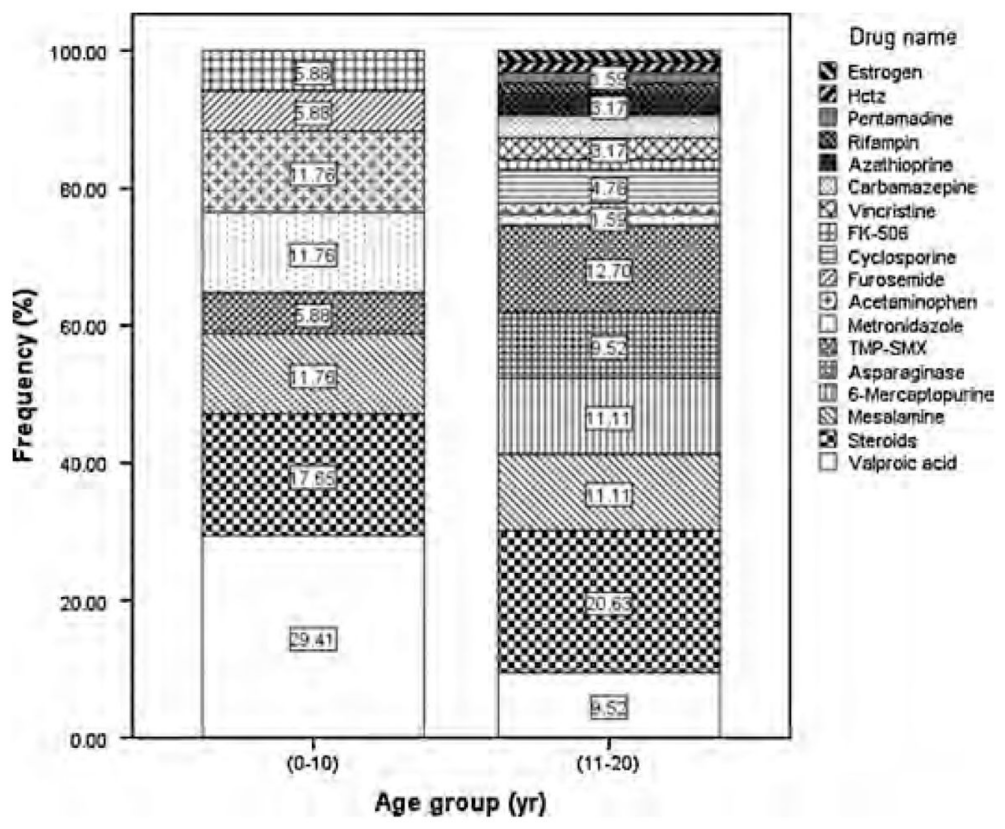

FIGURE 3.

Comparison of drugs associated with acute pancreatitis in younger ( $0-10$ years) vs older (11-20 years) children. *Indicates $P<0.05$ by Fisher exact probability test. TMP-SMX = trimethoprim/sulfamethoxazole. 


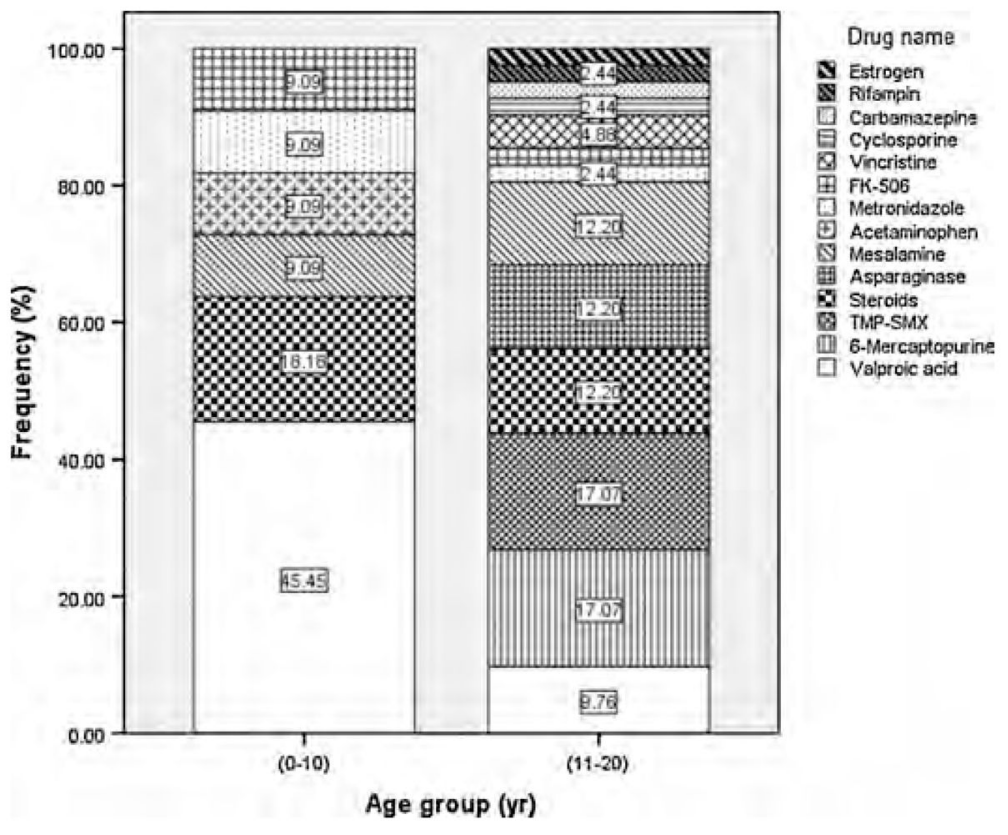

FIGURE 4.

Comparison of drugs associated with acute pancreatitis in younger vs older children who have no additional pancreatitis etiology. *Indicates $P<0.05$ by Fisher exact probability test. TMP-SMX = trimethoprim/sulfamethoxazole. 
TABLE 1

Characteristics of acute pancreatitis cases in children that were drug associated

\begin{tabular}{lcccc}
\hline & & \multicolumn{2}{c}{ Age subclassification, y } & \\
\cline { 2 - 4 } & Overall & $\mathbf{0 - 1 0}$ & $\mathbf{1 1 - 2 0}$ & $\boldsymbol{P}$ \\
\hline No. pancreatic episodes or cases & 55 & 16 & 38 & - \\
No. patients & 51 & 16 & 34 & - \\
Mean age, y (SD) & $12.8(5.3)$ & $5.9(2.9)$ & $15.8(2.7)$ & - \\
Male, \% (n) & $48.1(26)$ & $37.5(6)$ & $52.6(20)$ & 0.310 \\
BMI* & & & & \\
Median (IQR) & $49.1(13-86)$ & $49.1(0-71)$ & $54.9(14-88)$ & 0.254 \\
0-85th percentile, \% (n) & $86.0(37)$ & $90.9(10)$ & $87.5(28)$ & 0.807 \\
85th-95th percentile, \% (n) & $9.3(4)$ & $0.0(0)$ & $9.4(3)$ & 0.718 \\
>95th percentile, \% (n) & $4.7(2)$ & $9.1(1)$ & $3.1(1)$ & 1.000 \\
Weight for age & & & & \\
Median (IQR) & & & & \\
0-85th percentile, \% (n) & $85.2(46)$ & $87.5(14)$ & $86.8(33)$ & 0.708 \\
85th-95th percentile, \% (n) & $11.1(6)$ & $6.3(1)$ & $10.5(4)$ & 1.000 \\
>95th percentile, \% (n) & $3.8(2)$ & $6.3(1)$ & $2.6(1)$ & 0.888 \\
Ethnicity, \% (n) & & & & \\
White & $64.8(35)$ & $50.0(8)$ & $71.1(27)$ & 0.139 \\
Black & $16.7(9)$ & $25.0(4)$ & $13.2(5)$ & 0.425 \\
Hispanic & $11.1(6)$ & $18.8(3)$ & $7.9(3)$ & 0.493 \\
Other & $7.4(4)$ & $6.2(1)$ & $7.9(3)$ & 1.000 \\
\hline
\end{tabular}

$\mathrm{BMI}=$ body mass index $\mathrm{IQR}=$ interquartile range; $\mathrm{SD}=$ standard deviation.

* Adjusted for age and sex. 
TABLE 2

Comorbidities of acute pancreatitis cases in children that were drug associated, \% (n)

\begin{tabular}{lc}
\hline Seizure disorders & $20.0(11)$ \\
Acute lymphocytic leukemia & $20.0(11)$ \\
Crohn disease & $10.9(6)$ \\
Ulcerative colitis & $9.1(5)$ \\
Transplants/GVHD & $7.3(4)$ \\
HIV/AIDS & $3.6(2)$ \\
Asthma & $3.6(2)$ \\
Renal disorders & $3.6(2)$ \\
Acute myeloid leukemia & $3.6(2)$ \\
None & $10.9(6)$ \\
\hline
\end{tabular}

GVHD = graft-versus-host disease 
TABLE 3

Comparison of clinical presentation between acute pancreatitis cases in children that were drug associated versus non-drug associated *

\begin{tabular}{lccc}
\hline & \multicolumn{2}{c}{ Drug-associated pancreatitis } & \\
\cline { 2 - 3 } Symptoms, \% (n) & Yes & No & $\boldsymbol{P}$ \\
\hline Abdominal pain & $92.6(50)$ & $93.8(195)$ & 0.758 \\
Epigastric tenderness & $88.7(47)$ & $88.9(184)$ & 0.965 \\
Nausea/vomiting & $71.7(38)$ & $78.2(161)$ & 0.320 \\
\hline
\end{tabular}

* There was no statistically significant difference in clinical presentation between drug associated versus non-drug associated. 
TABLE 4

Comparison of radiographic modalities performed between acute pancreatitis cases in children that were drug associated versus non-drug associated

\begin{tabular}{lccl}
\hline & \multicolumn{2}{c}{ Drug-associated pancreatitis } & \\
\cline { 2 - 3 } & Yes & No & $P$ \\
\hline Any radiography performed, \% (n) & $87.3(48)$ & $81.9(177)$ & 0.347 \\
US, \% (n) & $67.3(37)$ & $68.1(147)$ & 0.912 \\
CT, \% (n) & $54.5(30)^{*}$ & $28.4(61)$ & 0.0002 \\
ERCP, \% (n) & $1.8(1)^{*}$ & $15.8(34)$ & 0.006 \\
MRCP, \% (n) & $0.0(0)$ & $5.1(11)$ & 0.128 \\
\hline
\end{tabular}

$\mathrm{CT}=$ computed tomography; $\mathrm{ERCP}=$ endoscopic retrograde cholangiopancreatography; $\mathrm{MRCP}=$ magnetic resonance cholangiopancreatography; US $=$ ultrasound

Indicates $P<0.01$ by comparing drug associated with non-drug associated. 
TABLE 5

Comparison of hospital management between acute pancreatitis cases in children that were drug associated vs non-drug associated

\begin{tabular}{lccc}
\hline & \multicolumn{2}{c}{ Drug-associated pancreatitis } & \\
\cline { 2 - 3 } & Yes & No & $P$ \\
\hline Median length of stay, d (IQR) & $10(4-19)^{*}$ & $4(3-8)$ & 0.0004 \\
GI consult ordered, \% (n) & $74.1(40)$ & $76.6(160)$ & 0.703 \\
NPO at admission, \% (n) & $88.9(48)$ & $93.7(193)$ & 0.243 \\
Mean time to nutrition after NPO, d (SD) & $2.1(1.9)$ & $2.2(1.7)$ & 0.763 \\
Modality of nutrition after NPO, \% (n) & & & \\
Oral, \% (n) & $58.3(28)^{\dagger}$ & $75.1(145)$ & 0.021 \\
Parenteral, \% (n) & $37.5(18)^{\dagger}$ & $21.2(41)$ & 0.019 \\
$\quad$ Enteral tube feedings, \% (n) & $4.2(2)$ & $3.6(7)$ & 1.000 \\
\hline
\end{tabular}

$\mathrm{IQR}=$ interquartile range NPO = nil per os; $\mathrm{SD}=$ standard deviation.

Indicates $P<0.001$ by Mann-Whitney test.

${ }^{\dagger}$ Indicates $P<0.05$ by $\chi^{2}$ analysis. 